\title{
Un rôle central, dans un environnement très concret
}

Paul Hoff

Correspondance:

Prof. Dr Paul Hoff

Clinique universitaire

psychiatrique de Zurich

Clinique pour la psychiatrie, la

psychothérapie et la psycho-

somatique

Lenggstrasse 31

Case postale 1931

CH-8032 Zurich

paul.hoff[at]puk.zh.ch
La relation entre une personne qui recherche de l'aide et celle qui détient les compétences et offre de l'aide joue un rôle fondamental depuis que la médecine existe. A tel point que cette question donne parfois l'impression d'être évidente et de s'expliquer d'ellemême; la réflexion qu'elle suscite paraît dénuée de lien direct avec le quotidien médical et menée pour ainsi dire dans un espace confiné et abstrait. Mais ce point de vue manque assurément de profondeur: comme toute expérience de vie, la relation médecinpatient voit le jour dans une situation concrète, en corrélation avec les personnes impliquées. En aucun cas elle ne se limite à des aspects techniques et de procédure comme la question de savoir comment obtenir une «traduction» optimale entre la description subjective du patient, empreinte de son vécu, et le langage nuancé du médecin, fondé sur des faits et des règles scientifiques. Par nécessité, il s'agit plutôt d'un sujet qui touche à des questions fondamentales, notamment à l'image que la médecine renvoie d'ellemême et à la répartition des rôles qui en découle en consultation et dans les processus thérapeutiques, mais aussi à des aspects plus théoriques comme les notions de maladie et de santé ou la valeur du diagnostic.

Dans les faits, ces questions se posent aujourd'hui à l'ensemble de la médecine [1, 2, 3, 4], mais c'est en psychiatrie et psychothérapie qu'elles apparaissent de

\section{פection of Depcbiatry.}

President-Dr. C. HUBert Bond, C.B.E.

\section{The Use and Abuse of the Relationship between Doctor and Patient in the Practice of Psychotherapy.}

\author{
By Mary C. Bell, M.B.
}

ANx understanding I may have upon this subject is mainly the outcome of observations made in my own hypnotic and analytical practice, of my own reactions to my analysts, and of my knowledge of the writings of psychoanalysts, but that does not mean that the subject is only of value from the psycho-analytical point of view. The relationship between doctor and patient is of primary importance in any psychotherapeutic practice, whether that of hypnotism, persuasion, re-education, or analysis.

The personality of the doctor as an asset in the building up of a successful practice is recognized in the term "a good bedside manner," and the man who

Une ancienne étude (1923) sur la relation médecin-patient en psychothérapie [9]. manière particulièrement frappante et récurrente. En effet, la personnalité du patient, sa manière de concevoir la relation avec autrui et l'impact d'éventuels troubles psychiques sur son histoire de vie représentent des éléments indispensables à tout diagnostic fiable et à un traitement durable $[5,6,7,8]$. Dès lors, le développement qui suit se réfère principalement à un point de vue psychiatrique, et donc à une pars pro toto de l'acte médical, mais il peut tout aussi bien être transposé à l'ensemble de la médecine.

\section{Médecin-patient: une relation «d'égal à égal»}

Nul ne peut mettre en doute (et ce également à l'ère de l'internet) la différence de savoir qui caractérise la relation entre le patient et le médecin, et qui ne représente que rarement un problème. Aujourd'hui, tout le monde s'accorde à dire que cette différence de connaissances ne doit pas créer de rapport hiérarchique: a priori, le médecin ne sait pas ce qui est mieux pour le patient, il n'a pas non plus compétence pour appréhender le comportement à venir, ni même la vie du patient. Au contraire, l'autorité du médecin repose sur des compétences médicales, constituées de son savoir et de sa capacité à personnaliser sa manière de transmettre ce savoir à chaque patient et à le convaincre. Pour cette démarche exigeante, il faut impérativement une relation solide.

Mettre en place une telle relation et la maintenir, éventuellement aussi sur une plus longue durée dans le cas de maladies chroniques, devient alors un véritable défi dont l'enjeu principal est de toujours essayer de garder l'équilibre entre proximité et distance, toutes deux nécessaires. En psychiatrie en particulier, il arrive souvent que des questions très personnelles soient évoquées dans le moindre détail; ce sont généralement des sujets que les patients ne sont prêts à aborder que dans leur environnement le plus proche. A cet égard, citons l'exemple de l'anamnèse biographique qui est au cœur même de l'acte médical en psychiatrie et psychothérapie. L'analyse de l'histoire de vie d'un patient est un défi à relever pour les deux parties; elle confronte le patient à la complexité de sa propre évolution, à des phases critiques de sa vie et à des événements à connotation négative comme la peur, la vexation et le doute. Le dialogue ne peut pas se poursuivre et être approfondi sans suffisamment de proximité personnelle et de confiance réciproque dans la relation médecin-patient. C'est aussi une des raisons principales pour lesquelles le secret médical 


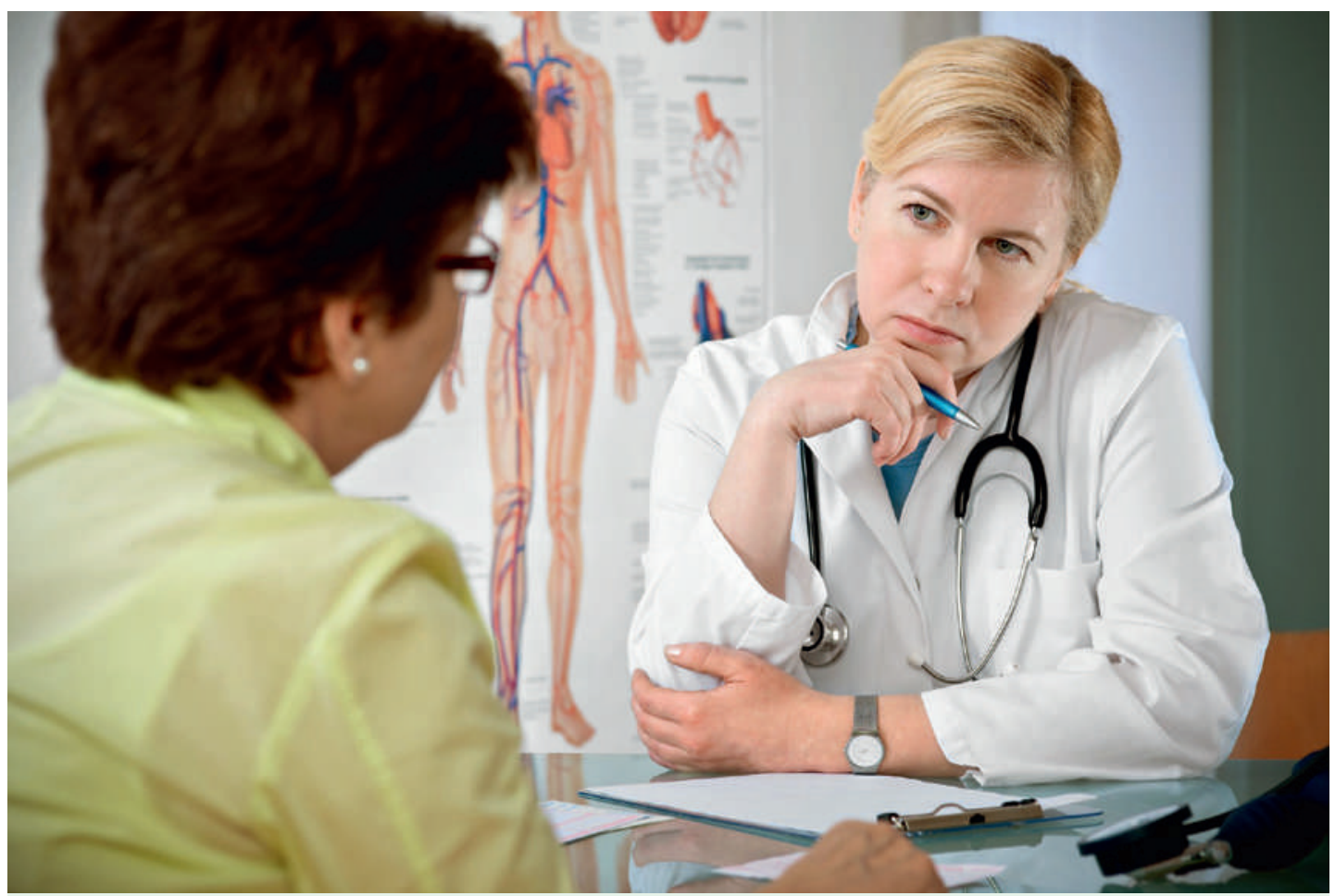

La relation entre le médecin et son patient constitue un élément extrêmement concret et tangible de notre profession.

se trouve depuis toujours au sommet de l'échelle des valeurs de la profession médicale.

\section{Risques possibles}

Pourquoi cet élément central de la relation médecinpatient soulève-t-il des questions aussi sensibles et délicates? Le mieux pour répondre à cette question est de considérer les deux extrémités de cette relation, la distance forcée et la proximité dénuée de sens critique.

\section{Un traitement efficace n'est pas conciliable} avec une empathie sans critique.

Si le médecin essaie de s'extraire le plus possible de la relation, il se voit comme un instrument de mesure objectif ou comme un catalyseur qui enclenche un processus de changement dans lequel il n'est absolument pas impliqué. Dans un contexte psychothérapeutique, cela risque d'irriter le patient et même de l'apeurer. Le patient souhaite une relation fondée sur un échange personnel avec son médecin; il n'entend pas répondre à des questions standard formulées d'avance ou simplement livrer des données pour compléter des échelles de diagnostics. Mais l'opposé, à savoir la proximité dénuée de sens critique, pose autant de problèmes. Sans aucun doute, le patient appréciera la volonté du médecin traitant d'écouter ses récits sans parti pris, d'essayer de les ressentir en y mettant une note personnelle et de la proximité. Mais si cette proximité devient un élément décisif ou l'unique élément porteur du traitement, elle perd alors ses effets. Il est certainement réconfortant et encourageant pour le patient de se sentir accepté, compris, et de voir que quelqu'un le prend en pitié. Pourtant, un traitement ne se résume pas à cette approche. En d'autres termes, un traitement efficace n'est pas conciliable avec une empathie sans critique.

Mais alors, comment résoudre ce dilemme? La dénomination initiale de notre profession, ars medica, souligne que l'art du médecin consiste à alimenter le dialogue en passant de la proximité empathique au regard critique sous-jacent, et vice-versa, en fonction de la situation. Grâce à ce mode de fonctionnement, il sera possible de personnaliser et de motiver les différentes étapes du traitement. Dans l'idéal, agissant pour ainsi dire de manière implicite, le médecin adoptera ce changement de perspective permanent sans que le patient ne soit impressionné par l'application d'une technique éprouvée. Pour bien cerner cette exigence élevée, il faut ajouter qu'une relation thérapeutique solide et durable ne peut exister qu'au-delà de toute distance technocrate et de toute fraternisation dénuée de sens critique.

Ce serait une erreur de penser que les aspects à risques de la relation médecin-patient interviennent uniquement lors de rares cas exceptionnels, ou même seulement lors de certains troubles psychiatriques. L'équilibre subtil, et adapté à chaque patient, entre 
proximité et distance appartient davantage à chaque entrevue médicale. Beaucoup de médecins, par exemple, se trouvent confrontés à des patients qui leur demandent un avis personnel sur tel ou tel problème, qui veulent connaître leur propre manière de mener leur vie ou de simples préférences personnelles; une démarche qui, prise séparément, ne remet nullement en cause la qualité de la relation thérapeutique ni ne s'approche d'un quelconque abus de cette relation. Néanmoins, le médecin doit faire preuve de tact pour réagir de manière appropriée à la question: «Comment gérez-vous ce problème dans la relation avec votre partenaire?» De manière appropriée signifie ici qu'il ne s'agit ni de donner l'impression au patient qu'il est rabroué ou qu'il n'est pas pris au sérieux, ni de l'embarquer dans une fraternisation apparente et hypocrite. Particulièrement dans le deuxième cas, dépasser la limite ne tient qu'à un fil pour ensuite ouvrir la porte à un abus. Si le médecin a mal assimilé la notion de proximité et qu'il répond à ce genre de questions par une description détaillée de sa situation personnelle, il induira une mauvaise répartition des rôles au détriment de l'efficacité du traitement et avec des effets préjudiciables pour le patient. Le caractère abusif de la situation est encore plus patent lorsque le traitement donne lieu à un lien étroit en privé, qui débouche ensuite sur une relation à caractère sexuel.

\section{Conclusion}

Dans tout acte médical, la relation avec le patient revêt une telle importance que nous devrions fournir tous les efforts pour l'évaluer et la faire évoluer en permanence, aussi bien du point de vue de sa définition que de notre comportement pratique. Loin d'être une notion abstraite dans un espace confiné, elle constitue un élément extrêmement concret et tangible de notre profession. Dès lors, idéaliser la relation médecin-patient ou la considérer comme une relation sacro-sainte, qui s'explique d'elle-même, devient contre-productif. A l'instar de toute autre forme d'actes médicaux, elle doit faire l'objet d'une réflexion critique selon une perspective scientifique mais aussi sous l'angle de la pratique. Dans le même temps, nous savons tous que des cas drastiques rappellent régulièrement à la mémoire des cercles spécialisés et de l'opinion publique le risque porté par tout abus, mais c'est à nous de garantir et de veiller à ce que ce risque, quelle que soit son importance, ne vienne pas interférer ou discréditer l'ensemble du débat que nous devons mener. A nous de lancer une réflexion critique, transparente et pratique autour de la relation médecin-patient, en favorisant un échange continu, loin de toute idéalisation naïve et de la défiance générale. C'est - à juste titre - ce que les patients attendent de nous.

\section{Références}

1 Begenau J, Schubert C, Vogd W (Hrsg.). Die ArztPatient-Beziehung. Stuttgart: Kohlhammer; 2010.

2 Deter H C (Hrsg.) (2010). Die Arzt-Patient-Beziehung in der modernen Medizin. Vandenhoeck \& Ruprecht, Göttingen.

3 Eveleigh R M, Muskens E, van Ravesteijn H, van Dijk I, van Rijswijk E, Lucassen P. An overview of 19 instruments assessing the doctor-patient relationship: different models or concepts are used. J Clin Epidemiol. 2012. 65:10-5.

4 Hurrelmann K, Leppin A (Hrsg) (2001). Moderne Gesundheitskommunikation. Vom Aufklärungsgespräch zur E-Health. Bern: Huber; 2001.

5 Boothe B. Das Narrativ. Biografisches Erzählen im psychotherapeutischen Prozess. Stuttgart: Schattauer; 2011

6 Hoff P. Psychiatrie: Ein Blick von innen. Geschichte, Theorien, Fälle. Oberhofen am Thunersee: Zytglogge; 2011.

7 Michel K, Jobes DA (Hrsg.). Building a Therapeutic Alliance With the Suicidal Patient. Washington DC: American Psychological Association; 2011.

8 Scharfetter C. Was weiss der Psychiater vom Menschen? Bern: Huber; 2010.

9 Bell MC. The Use and Abuse of the Relationship between Doctor and Patient in the Practice of Psychotherapy. Proc R Soc Med. 1923.16 (Sect Psych):12 -20. 